\title{
Validação transcultural da Escala CONNECT - Uma medida de continuidade do cuidado em serviços de saúde mental
}

\author{
Cross-cultural validation of the Portuguese version (Brazil) \\ CONNECT - A care continuity as mental health services
}

Diego de Lima Fonseca', Michelli Melo Grama', Giovanni Marcos Lovisi', Lúcia Abelha’

\section{RESUMO}

Objetivo: Realizar validação transcultural da versão em português (Brasil) da CONNECT - Uma medida de continuidade do cuidado em serviços de saúde mental, em uma amostra de 150 indivíduos com transtornos mentais graves. Métodos: A validação de constructo foi realizada utilizando-se a Análise de Componentes Principais (ACP). A concordância foi determinada pelo Coeficiente de Correlação Intraclasse (ICC). Também foi realizada a análise de correlação entre os itens da escala, as subescalas e a escala geral. Resultados: Os entrevistados tinham idade média de 48,91 anos, sendo maioria homens (60\%), diagnosticados com esquizofrenia (70,7\%). O ICC encontrado foi bom ou quase perfeito em 7 das 9 subescalas e a correlação entre as subescalas e a escala geral foi significativa em todas as subescalas testadas. A ACP ex-

\section{Palavras-chave}

Estudo de validação, continuidade da assistência ao paciente, serviços de saúde mental.

\section{Keywords}

Validation study, continuity of patient care, mental health services. plicou 66,29\% da variância de toda a escala por meio de nove fatores. Conclusão: A ACP confirmatória executada apresenta uma estrutura correspondente à estrutura original da escala. Até o momento a CONNECT é o único instrumento de avaliação da continuidade do cuidado desses pacientes que está adaptado ao contexto brasileiro. Os resultados obtidos neste estudo mostram que a versão Brasileira da Escala CONNECT pode ser utilizada com segurança.

\section{ABSTRACT}

Objective: Perform cross-cultural validation of the Portuguese version (Brazil) CONNECT - A care continuity as mental health services, in a sample of 150 individuals with severe mental disorders. Methods: A construct validation was performed using the Principal Component Analysis (PCA). The agreement was determined by Intraclass Correlation Coefficient (ICC). It was also performed a correlation analysis between the items of the scale, subscales and general scale. Results: Respondents had an average age of 48.91 years, most were men (60\%) diagnosed with schizophrenia (70.7\%). The ICC found was good and almost perfect in 7 of the 9 subscales, the correlation between subscales and the overall scale was significant in all tested subscales. The PCA explained $66.29 \%$ of the variance of all scale through nine factors. Conclusion: PCA demonstrated a structure corresponding to the original structure of the scale. By the time the CONNECT is the only assessment tool of continuity of care of these patients is adapted to the Brazilian context. The results of this study show that the Brazilian version of CONNECT scale can be used safely.

1 Universidade Federal do Rio de Janeiro (UFRJ), Instituto de Estudos em Saúde Coletiva.
Recebido em

$18 / 1 / 2017$
Endereço para correspondência: Diego de Lima Fonseca

Instituto de Estudos em Saúde Coletiva, Universidade Federal do Rio de Janeiro

Praça Jorge Machado Moreira, Ilha do Fundão, Cidade Universitária

21944-970 - Rio de Janeiro, RJ, Brasil

Telefone: +55 (21) 99479-6922

E-mail: diegofonseca@iesc.ufrj.br 


\section{INTRODUÇÃO}

A reforma da assistência psiquiátrica iniciou-se na década de 1970 no Brasil, levando à redução acentuada do número de leitos e de hospitais psiquiátricos, não havendo uma correspondente implantação de uma rede de atenção inserida na comunidade para suprir a demanda. Essa rede, que se instalou tardiamente, tem como base estratégica os Centros de Atenção Psicossocial (CAPS), assim como os Serviços Residenciais Terapêuticos (SRT), as unidades psiquiátricas em hospitais gerais, as ações de saúde mental na atenção básica e os Programas de Volta para Casa e de inclusão social pelo trabalho ${ }^{1-4}$.

Com esse novo direcionamento para a atenção na comunidade, a continuidade do cuidado tem sido considerada um elemento essencial para o planejamento de ações e serviços para os pacientes crônicos, tais como os indivíduos com transtornos mentais graves e persistentes. Isso ocorre pelo fato de que esses pacientes utilizam diversos serviços ao longo de suas vidas, sendo esses serviços um resultado da combinação de fatores tais como acesso adequado ao cuidado, habilidades dos profissionais, bom fluxo de informação, entendimento entre técnicos e serviços e boa coordenação e integração entre profissionais para manter a consistência da atenção ${ }^{5}$.

Três estudos se destacam na análise conceitual de continuidade do cuidado em indivíduos com transtornos mentais graves e persistentes, sendo referências para os pesquisadores que avaliam a continuidade do cuidado em serviços de saúde mental e/ou desenvolvem medidas para operacionalizá-la. São eles: os estudos de Bachrach ${ }^{6}$, Tessler et al. ${ }^{7}$ e Test ${ }^{8}$. Outro estudo realizado em 2004 tentou operacionalizar esse construto por meio de uma revisão sistemática e entrevistas com familiares e pacientes?.

O estudo de Test ${ }^{8}$ assinala a relevância da continuidade transversal e de um sistema de cuidado abrangente e integrado, e destaca as principais causas da descontinuidade do cuidado: falhas no sistema de informações e de gerenciamento; falta de coordenação dos serviços, de controle de tratamento, de comunicação ou responsabilidades claramente definidas; e dificuldades em manter o paciente engajado ao serviço, deixando, porém, de abarcar outras dimensões trabalhadas pelos demais autores.

Tessler et al.? ressaltam a importância da disponibilidade de todo o tratamento ao longo do tempo, para que o paciente possa ter acesso ao cuidado que necessita, sendo a descontinuidade uma possibilidade, chegando ao abandono do tratamento, quando algum elemento essencial do cuidado for ausente ou quando existirem falhas na coordenação ou comunicação. O autor propõe um conceito de continuidade do cuidado com três dimensões: planejamento de alta; transferência rápida e bem-sucedida entre os serviços, indicada pelo tempo entre a alta hospitalar e o primeiro atendi- mento no serviço referenciado; e planejamento de serviços individualizados.

Bachrach $^{6}$ realizou um dos mais amplos e mais citados estudos, definindo continuidade do cuidado em saúde mental como o fluxo ordenado e ininterrupto dos pacientes entre os diversos elementos do sistema de prestação de serviço. Identificou sete dimensões necessárias para assegurar a continuidade do cuidado: longitudinal, individual, transversal, acessibilidade, flexibilidade, relacional e comunicação.

Nesse mesmo estudo o autor aponta que essa continuidade deve iniciar tão logo haja a entrada do paciente no serviço de saúde, seja no nível primário, secundário ou terciário, e terminar somente quando as necessidades do paciente forem cessadas (Dimensão longitudinal); o cuidado deve ser voltado para o paciente e sua família, de maneira individual (Dimensão individual), e ser ofertado por uma ampla variedade de serviços de acordo com as necessidades específicas de cada paciente (Dimensão transversal), sendo flexível aos seus progressos e/ou declínios (Flexibilidade); o acesso aos serviços de saúde precisa ser garantido e facilitado (Acessibilidade); os serviços precisam estar integrados, de maneira que possam se comunicar e trocar informações sobre os pacientes, facilitando assim o cuidado destes (Relacional); e as relações entre os pacientes e o serviço/equipe precisam ser estreitadas e aprofundadas (Comunicação).

O reconhecimento da importância da continuidade do cuidado e de seu amplo conceito tem levado ao desenvolvimento crescente de instrumentos de medida para diferentes grupos de pacientes, incluindo-se aí os pacientes com transtornos mentais graves. A CONNECT é uma ferramenta de avaliação da continuidade do cuidado em serviços de saúde mental designada para pacientes com transtornos mentais graves e persistentes e serve como uma indicadora da qualidade do atendimento nesses serviços. Mede a interação e o relacionamento entre profissionais e usuários dos serviços de saúde mental, considerando as atividades diárias de fornecimento e recebimento de cuidados.

A escala foi desenvolvida nos Estados Unidos em $2003^{10}$ por Ware et al. (Mental Health Services Research) como um instrumento de medida formal que reflete as perspectivas dos usuários dos serviços de saúde mental, com o objetivo de apoiar e fortalecer as pesquisas sobre continuidade do cuidado nesses serviços. Escrita originalmente na língua inglesa, seus domínios conceituais foram definidos por meio de um estudo etnográfico realizado entre 1996 e 1997 por esse mesmo grupo de pesquisadores. Sua versão original em inglês demonstrou atender aos critérios de confiabilidade, validade e consistência interna. Essa escala foi adaptada ao espanhol em $2007^{11}$ por Chavez et al., apresentando resultados positivos semelhantes aos da versão original em inglês. Quando já existe um instrumento validado em outro país para o mesmo tipo de medida que se deseja fazer, que tenha qualidades psicométricas e seja relevante, o mais ade- 
quado é que seja realizada validação de uma versão adaptada transculturalmente do instrumento, devido a diversos fatores, incluindo a comparação dos dados entre diferentes países $^{12}$

O Brasil vem construindo uma rede de atenção de serviços de saúde mental com base comunitária para indivíduos com transtornos mentais graves desde a década de 1980, sendo o primeiro CAPS instalado em 1986 na cidade de São Paulo. Nesse sentido, medir o quanto essa rede permite a continuidade do tratamento é de grande interesse, ainda mais para esses indivíduos que apresentam suscetibilidade ao abandono de tratamento por diversos fatores que envolvem seus transtornos mentais e suas próprias histórias de vida ${ }^{13-15}$

No Brasil, não é comum a medição e a avaliação da continuidade do cuidado nos serviços de saúde mental, o que pode ser devido à inexistência de um instrumento de medida construído ou adaptado e validado para a realidade brasileira. Portanto, o objetivo do presente estudo é validar a versão brasileira da escala CONNECT.

\section{MÉTODOS}

As etapas anteriores ao estudo de validação incluem a adaptação transcultural do instrumento com tradução, retrotradução e painel de especialistas e a avaliação de confiabilidade desse com a realização de um pré-teste na aplicação da metodologia de teste e reteste com intervalo médio de 15 dias. Essas etapas foram realizadas pelo mesmo grupo de pesquisadores, que demonstraram a confiabilidade da versão em português do instrumento ${ }^{16}$.

\section{Local do estudo}

A população do estudo foi composta por pacientes que estavam em tratamento nas seguintes unidades de atendimento em saúde mental:

- Instituto de Psiquiatria da Universidade Federal do Rio de Janeiro (IPUB/UFRJ). Localizado no campus da Praia Vermelha da UFRJ, as suas instalações contam com duas enfermarias (feminina e masculina), ambulatório de adultos, infanto-juvenil e hospital-dia para adultos, além de três unidades de moradia assistida fora do campus. As duas enfermarias somam 117 leitos psiquiátricos e o hospital-dia possuía cerca de 140 pacientes matriculados.

- CAPS I da cidade de Guapimirim, que está localizada na Serra dos Órgãos, integrando a região metropolitana do Estado do Rio de Janeiro. A população do município segundo o Instituto Brasileiro de Geografia e Estatística (IBGE) é de 51.483 habitantes no último censo, em 2010. São atendidos atualmente cerca de 70 pacientes ${ }^{17}$.
- CAPS II da cidade de Carmo, que está localizada no centro fluminense, fazendo divisa com o Estado de Minas Gerais, com população de cerca de 19.000 habitantes, divididos em $320 \mathrm{~km}^{2}$. Estão vinculadas ao CAPS 21 residências terapêuticas, que abrigam os ex-internos do hospital da cidade. São 157 profissionais atuando diretamente no CAPS ou nas residências terapêuticas

\section{População do estudo}

- O tamanho da amostra recomendado para validação de instrumentos pela Organização Mundial da Saúde - OMS (WHO, 2012) é de cinco indivíduos para cada item do instrumento. A escala CONNECT geral possui no total 37 itens. Os demais se referem aos itens da escala de transição, sendo aplicáveis apenas aos indivíduos que mudaram o atendimento de serviços nos últimos seis meses, os quais em geral são poucos. No estudo de validação da versão em espanhol, o percentual de indivíduos que responderam às escalas de transição foi de 6,7\% para transição internação-ambulatório, 15,3\% para mudança de psiquiatra, $6 \%$ para técnico de referência, 16,7\% para mudança de residência e $24,7 \%$ para serviços de emergência"1. Portanto, o tamanho definido para a amostra do presente estudo foi de 150 indivíduos.

\section{Critérios de inclusão}

- Indivíduos maiores de 18 anos;

- Indivíduos com diagnóstico de transtorno mental grave de acordo com a CID-10: F20-22; F24-29; F30-31; F32.3; F33.3. Para este estudo foi definido que um indivíduo com transtorno mental grave e persistente é aquele que sofre de transtorno psiquiátrico grave de longo prazo, com um grau variável de deficiência e disfunção social. Tal definição se baseia em três critérios: diagnóstico de psicose não orgânica ou transtorno de personalidade; história de transtorno mental ou duração de tratamento maior ou igual a dois anos.

\section{Critérios de exclusão}

- Pacientes que se recusassem a fazer a entrevista e a assinar o termo de consentimento livre e esclarecido.

- Pacientes com incapacidade de responder às perguntas, como, por exemplo, pacientes que apresentassem retardo mental, problemas de linguagem ou que estivessem em fase aguda do transtorno mental.

\section{Descrição do instrumento}

A CONNECT é um instrumento de medida da continuidade do cuidado em serviços de saúde mental para indivíduos com transtorno mental grave e persistente e foi desenvolvido nos Estados Unidos em 2003 por Ware et al. ${ }^{10}$. A versão 
brasileira do instrumento foi realizada pelo mesmo grupo de pesquisadores, seguindo as recomendações da OMS, e manteve a estrutura original da escala. A medida é feita por meio de uma entrevista com os pacientes, utilizando um questionário de respostas fixas, com 13 escalas com 57 itens distribuídos entre elas e um indicador de um único item, agrupados dentro de cinco domínios: conhecimento, flexibilidade, disponibilidade, coordenação e transições. Apenas 34 itens são aplicados a todos os indivíduos. O domínio Conhecimento é abordado pelas subescalas "Conhecimento do Psiquiatra" e "Conhecimento do Técnico de Referência", cada uma com seis itens, totalizando 12 itens nesse domínio, e visa verificar o quanto o paciente sente que os profissionais sabem a respeito de sua condição/necessidades.

O domínio Disponibilidade - "Disponibilidade do Técnico de Referência" e "Disponibilidade do Psiquiatra" -, cada um com três itens, totalizando seis itens, visa verificar o quanto o paciente consegue contato com os profissionais listados fora do ambiente de consulta.

O domínio Flexibilidade envolve apenas uma subescala "Flexibilidade de Psiquiatra/Técnico de Referência", com seis perguntas sobre a flexibilidade dos profissionais para alteração de agendas de consulta.

O domínio Coordenação envolve as subescalas "Coordenação do Psiquiatra/Técnico de Referência" e "Médico Clínico", além do item único "Coordenação Geral", compostas por 3, 2 e 1 item, respectivamente.

O domínio Transição envolve "Transição Internação-Ambulatório", "Transição do Serviço de Emergência", "Mudança de Psiquiatra", "Mudança de Técnico de Referência", "Mudança de Domicílio". São esses 23 itens de transição que só se aplicam a pacientes que tenham experimentado essas transições no último ano e visam mensurar o quanto o serviço conseguiu atuar para minorar os efeitos dessas transições.

\section{Aplicação do instrumento}

As entrevistas foram realizadas individualmente por dois pesquisadores em Saúde Coletiva do Instituto de Estudos em Saúde Coletiva da Universidade Federal do Rio de Janeiro (IESC/UFRJ), ambos com experiência prévia de aplicação do instrumento. As entrevistas foram realizadas em local privado e durante o horário de funcionamento de cada serviço, tendo duração aproximada de 20 minutos.

\section{Análises estatísticas}

Foram calculadas estatísticas descritivas em relação às variáveis sociodemográficas e às categorias diagnósticas. A média e o desvio-padrão (DP) também foram usados para descrever a amostra. A média das subescalas foi calculada considerando o percentual da pontuação máxima atingida, conforme preconizado pelos demais estudos com a escala original. Essa estratégia permite a comparação entre elas, sendo controlado o efeito do número de itens em cada uma.
A concordância foi determinada pelo Coeficiente de Correlação Intraclasse (ICC). Neste estudo, para interpretação do ICC, foram adotados os valores sugeridos por McDowell ${ }^{18}$, sendo: fraca ou pobre concordância ( $\leq 40)$; moderada concordância (41 - 60); boa ou substancial concordância (61 80) e muito boa ou quase perfeita concordância (> 81).

Também foi calculada a correlação entre as subescalas e a escala completa, por meio do coeficiente de correlação de Pearson. Para Cohen ${ }^{19}$, valores entre 0,10 e 0,29 podem ser considerados pequenos; escores entre 0,30 e 0,49 podem ser classificados como médios; e valores entre 0,50 e 1 podem ser interpretados como grandes.

A estrutura interna do questionário foi testada por meio da sua reprodução empírica usando a Análise de Componentes Principais (ACP). Foi utilizada uma solução com o número de nove fatores coincidindo com o número de subescalas da CONNECT, excluindo-se as escalas de transição, usando a ACP e rotação ortogonal (Varimax).

O banco de dados foi criado em uma máscara de entrada no Programa EPI-INFO 3.5.1 e analisado nos pacotes estatísticos Statistical Package for the Social Sciences (SPSS) 16.0 e PEPI-for-Windows (WinPepi).

\section{Considerações éticas}

O presente estudo foi submetido ao Comitê de Ética e Pesquisa (CEP) do Instituto de Estudos em Saúde Coletiva (IESC/ UFRJ), tendo sido aprovado (CAAE 16047713.5.0000.5286). As direções das instituições participantes concordaram com a realização da pesquisa.

Os entrevistados foram informados de que sua não participação não influenciaria no tratamento recebido e que poderiam interromper a participação a qualquer momento. O acesso ao banco de dados ficará restrito aos pesquisadores do estudo, sendo garantido aos participantes o sigilo e a confidencialidade dos dados.

\section{RESULTADOS}

Os indivíduos entrevistados tinham idade entre 18 e 86 anos, com média de 48,91 anos ( $D P=12,35)$. Em relação ao sexo, 60 (40\%) eram mulheres. Os entrevistados, em sua maioria, eram solteiros (69,3\%), tanto em relação aos homens $(77,4 \%)$ quanto em relação às mulheres $(57,9 \%)$. O nível educacional variou bastante dentre os entrevistados, sendo mais frequente o ensino fundamental incompleto/completo (53,3\%) e médio, incompleto/completo (30,7\%). Entre os entrevistados, 53,3\% ( $n=80$ ) eram do Hospital-dia do IPUB, 25,3\% ( $n=$ 38) eram dos APS de Guapimirim e $21,3 \%(n=32)$, dos CAPS de Carmo. A esquizofrenia (F20.X) foi a categoria diagnóstica mais frequente dentre os entrevistados $(71,4 \%)$.

Observa-se ainda que os entrevistados nos três locais da pesquisa apresentaram características sociodemo- 
gráficas diferentes estatisticamente $(p<0,05)$. O CAPS de Guapimirim apresentou o grupo mais jovem, mulheres (57,9\%), enquanto em Carmo predominou idosos do sexo masculino $(87,5 \%)$. Nos três locais houve predomínio de solteiros, sendo mais marcante em Carmo (87,5\%). No tocante ao nível educacional, o grupo do IPUB apresentava a maior escolaridade, seguida de Guapimirim, com Carmo apresentando o menor nível de escolaridade. Quanto à CID dos entrevistados, embora também ocorra diferença significativa, percebe-se que nos três locais a esquizofrenia foi o transtorno mental mais frequente, sendo maior no CAPS de Guapimirim (83,8\%), seguida pelo transtorno afetivo bipolar aparecendo com frequência maior no Hospital-dia do IPUB $(26,9 \%)$ (Tabela 1).

Do total de oito subescalas que poderiam ser analisadas, excluiu-se a escala com apenas uma questão, já que a análise não pode ser aplicada com apenas uma variável. Dessas, duas apresentaram correlação quase perfeita, cinco tiveram correlação boa ou substancial e duas mostraram correlações moderadas (Tabela 2).
A Coordenação Geral foi a subescala que obteve a maior pontuação, com 89,83\% da pontuação máxima atribuída. A subescala Transição Internação-Ambulatório teve a segunda maior pontuação, registrando 83,20\% da pontuação total.

As subescalas Coordenação do Psiquiatra/Técnico de Referência, Transição do Serviço de Emergência, Mudança de Técnico de Referência, Flexibilidade do Psiquiatra/Técnico de Referência e Mudança de Psiquiatra obtiveram, respectivamente, os seguintes percentuais: 66\%, 65,06\%, 65\%, 61,9\% e $54,91 \%$, denotando uma aderência de moderada a boa. Em relação à consistência interna da escala, apenas uma apresentou a baixo (Disponibilidade do Psiquiatra). As demais apresentaram a de moderado a muito alto (Tabela 3).

A correlação entre as subescalas e a escala completa foi moderada a alta, sendo significativa em todas as subescalas centrais aplicadas e em duas das cinco subescalas de transição. As correlações foram menores entre as subescalas. Destaca-se a correlação alta entre as subescalas Conhecimento do Técnico de Referência e Mudança de Técnico de Referência $(0,891, \mathrm{p}<0,01)$ (Tabela 4).

Tabela 1. Características sociodemográficas e categorias diagnósticas da população estudada distribuídas por locais da realização da pesquisa

\begin{tabular}{|c|c|c|c|c|c|c|c|}
\hline Variáveis & Categorias & $\mathrm{n}$ & $\%$ & Guapimirim (\%) & IPUB (\%) & Carmo (\%) & Valor dep \\
\hline \multirow[t]{2}{*}{ Sexo } & Feminino & 60 & 40,0 & 57,9 & 42,5 & 12,5 & $<0,01$ \\
\hline & Masculino & 90 & 60,0 & 42,1 & 57,5 & 87,5 & \\
\hline \multirow[t]{4}{*}{ Faixa etária } & $\leq 30$ anos & 13 & 8,7 & 25,8 & 3,5 & 6,1 & $<0,01$ \\
\hline & 31 a 40 anos & 23 & 15,3 & 19,4 & 16,3 & 9,1 & \\
\hline & 41 a 50 anos & 44 & 29,3 & 22,6 & 34,9 & 21,2 & \\
\hline & $\geq 51$ anos & 70 & 46,7 & 32,3 & 45,3 & 63,6 & \\
\hline \multirow[t]{4}{*}{ Estado civil } & Solteiro & 104 & 69,3 & 65,8 & 63,8 & 87,5 & 0,04 \\
\hline & Casado/União estável & 17 & 11,3 & 10,5 & 12,5 & 9,4 & \\
\hline & Separado/Divorciado & 23 & 14,3 & 13,2 & 21,3 & 3,1 & \\
\hline & Viúvo & 6 & 4,0 & 10,5 & 2,5 & 0,0 & \\
\hline \multirow[t]{4}{*}{ Nível educacional } & Analfabeto & 11 & 7,3 & 13,2 & 1,3 & 15,6 & $<0,01$ \\
\hline & Nível fundamental incompleto/Completo & 80 & 53,3 & 68,4 & 35,0 & 81,3 & \\
\hline & Ensino médio incompleto/Completo & 46 & 30,7 & 15,8 & 48,8 & 3,1 & \\
\hline & Nível superior incompleto/Completo & 13 & 8,7 & 2,6 & 15,1 & 0,0 & \\
\hline \multirow[t]{10}{*}{ Categorias diagnósticas } & Esquizofrenia (F20.x) & 107 & 71,4 & 83,8 & 67,9 & 78,2 & $<0,01$ \\
\hline & Transtornos delirantes persistentes (F22.x) & 3 & 2,0 & 3,2 & 0,0 & 0,0 & \\
\hline & Esquizofrenia (F20.x) & 107 & 71,4 & 83,8 & 67,9 & 78,2 & $<0,01$ \\
\hline & Transtornos delirantes persistentes (F22.x) & 3 & 2,0 & 3,2 & 0,0 & 0,0 & \\
\hline & Transtorno esquizoafetivo do tipo maníaco (F25.0) & 1 & 0,7 & 6,6 & 0,0 & 0,0 & \\
\hline & Psicose não orgânica não especificada (F29) & 2 & 1,3 & 3,2 & 0,0 & 3,1 & \\
\hline & Episódio maníaco (F30.x) & 6 & 4,0 & 0,0 & 3,9 & 3,1 & \\
\hline & Transtorno afetivo bipolar (F31.x) & 28 & 18,6 & 0,0 & 26,9 & 9,4 & \\
\hline & Depressão grave com sintomas psicóticos (F32.3) & 1 & 0,7 & 3,2 & 0,0 & 3,1 & \\
\hline & $\begin{array}{l}\text { Transtorno depressivo recorrente, episódio atual } \\
\text { grave com sintomas psicóticos (F33.3) }\end{array}$ & 2 & 1,3 & 0,0 & 1,3 & 3,1 & \\
\hline
\end{tabular}


Tabela 2. Concordância para os escores das subescalas com o escore total da CONNECT (ICC - IC 95\%)

\begin{tabular}{|c|c|c|c|}
\hline Subescalas & Interpretação de concordância & $\mathrm{N}^{\circ}$ de itens & ICC (IC 95\%) \\
\hline Conhecimento do Psiquiatra & Quase perfeita & 6 & $0,829(0,783-0,869)$ \\
\hline Conhecimento do Técnico de Referência & Quase perfeita & 6 & $0,849(0,808-0,885)$ \\
\hline Disponibilidade do Psiquiatra & Moderada & 3 & $0,441(0,266-0,580)$ \\
\hline Disponibilidade do Técnico de Referência & Boa ou substancial & 3 & $0.613(0,489-0,711)$ \\
\hline Psiquiatra/Técnico de referência/Profissional do CAPS & Boa ou substancial & 7 & $0,657(0,567-0,735)$ \\
\hline Flexibilidade do Psiquiatra/Técnico de Referência & Boa ou substancial & 6 & $0,707(0,625-0,775)$ \\
\hline Coordenação do Psiquiatra/Técnico de Referência & Boa ou substancial & 3 & $0,725(0,637-0,794)$ \\
\hline Coordenação Geral & NA & 1 & NA \\
\hline Médico Clínico & Boa ou substancial & 2 & $0,776(0,691-0,838)$ \\
\hline
\end{tabular}

NA: não se aplica (não foi possível calcular o ICC em funçăo de a escala ter só 1 item).

Tabela 3. Escore médio, desvio-padrão (DP) e consistência interna (alfa de Cronbach) por subescala para todos os itens da CONNECT

\begin{tabular}{|c|c|c|c|c|}
\hline Subescalas da CONNECT & Número de itens & Escore médio (\%) & Desvio-padrão (\%) & Alpha de Cronbach \\
\hline Conhecimento do Psiquiatra & 6 & 45,56 & 27,92 & 0,83 \\
\hline Conhecimento do Técnico de Referência & 6 & 44,91 & 30,25 & 0,85 \\
\hline Disponibilidade do Psiquiatra & 3 & 20,78 & 23,38 & 0,44 \\
\hline Disponibilidade do Técnico de Referência & 3 & 34,08 & 29,43 & 0,61 \\
\hline Psiquiatra/Técnico de referência/Profissional do CAPS & 7 & 24,19 & 19,18 & 0,66 \\
\hline Flexibilidade do Psiquiatra/Técnico de Referência & 6 & 61,90 & 28,01 & 0,71 \\
\hline Coordenação do Psiquiatra/Técnico de Referência & 3 & 66,10 & 33,51 & 0,72 \\
\hline Coordenação Geral & 1 & 89,83 & 23,07 & NA \\
\hline Médico Clínico & 2 & 40,08 & 16,53 & 0,78 \\
\hline Escala Completa & 34 & - & - & - \\
\hline
\end{tabular}

NA: não se aplica (não foi possível calcular 0 alpha de (ronbach em função de a escala só ter só 1 item).

Tabela 4. Coeficiente de correlação de Pearson entre as subescalas e a escala CONNECT

\begin{tabular}{|c|c|c|c|c|c|c|c|c|c|c|c|}
\hline & & \multicolumn{10}{|c|}{ Subescalas } \\
\hline & & CONNECT & 1 & 2 & 3 & 4 & 5 & 6 & 7 & 8 & 9 \\
\hline & CONNECT & 1 & $0,688^{* *}$ & $0,790^{* *}$ & $0,403^{* *}$ & $0,558^{* *}$ & $0,611^{* *}$ & $0,678^{* *}$ & $0,571^{* *}$ & $0,316^{* *}$ & $0,244^{* *}$ \\
\hline 1 & Conhecimento do Psiquiatra & & 1 & $0,545^{* *}$ & $0,318^{* *}$ & $0,175^{*}$ & $0,281^{* *}$ & $0,369^{* *}$ & $0,289^{* *}$ & $0,237^{* *}$ & 0,021 \\
\hline 2 & Conhecimento do Técnico de Referência & & & 1 & $0,214^{* *}$ & $0,403^{* *}$ & $0,415^{* *}$ & $0,393^{* *}$ & $0,346^{* *}$ & $0,221^{* *}$ & 0,049 \\
\hline 3 & Disponibilidade do Psiquiatra & & & & 1 & $0,256^{* *}$ & $0,255^{* *}$ & 0,104 & 0,009 & 0,003 & $-0,022$ \\
\hline 4 & Disponibilidade do Técnico de Referência & & & & & 1 & $0,388^{* *}$ & $0,343^{* *}$ & $0,241^{* *}$ & 0,120 & 0,132 \\
\hline 5 & Psiquiatra/Técnico de Referência/Profissional do CAPS & & & & & & 1 & $0,298^{* *}$ & $0,240^{* *}$ & 0,101 & 0,099 \\
\hline 6 & Flexibilidade do Psiquiatra/Técnico de Referência & & & & & & & 1 & $0,453^{* *}$ & $0,288^{* *}$ & $0,204^{*}$ \\
\hline 7 & Coordenação do Psiquiatra/Técnico de Referência & & & & & & & & 1 &, $246^{* *}$ & 0,124 \\
\hline 8 & Coordenação Geral & & & & & & & & & 1 & $-0,023$ \\
\hline 9 & Médico Clínico & & & & & & & & & & 1 \\
\hline
\end{tabular}

* Correlações significativas com valor de $p \leq 0,05$ (bicaudal).

** Correlações significativas com valor de $p \leq 0,01$ (bicaudal).

A ACP foi conduzida nos 37 itens do instrumento que foram aplicados a todos os indivíduos. A medida de Kaiser-Meyer-Olkin verificou a adequação amostral para a análise $(\mathrm{KMO}=0,743)$. O teste de esfericidade de Bartlett (qui-quadrado $=2727,408, p<0,001)$ indicou que as correlações entre os itens são suficientes para a realização da ACP.
A ACP explicou 66,29\% da variância de toda a escala por meio dos nove fatores. A correlação entre os fatores gerados pela análise dos componentes principais e as subescalas originais se mostrou relevante. A subescala Conhecimento do Psiquiatra revelou-se mais relacionada ao fator $2(p=0,658)$, enquanto a subescala Conhecimento do 
Técnico de Referência mostrou-se mais relacionada ao fator 1 $(p=0,899)$.

As subescalas Disponibilidade do Psiquiatra e Disponibilidade do Técnico de Referência apresentaram forte correlação com o mesmo fator ( $p=0,748$ e $p=0,561$ ) respectivamente.

A subescala Psiquiatra/Técnico de Referência/Profissional do CAPS mostrou-se mais relacionada ao fator $3(p=0,647)$. A subescala Flexibilidade de Psiquiatra/Técnico de Referência revelou-se mais relacionada ao fator $5(p=0,801)$.

A subescala Coordenação do Psiquiatra/Técnico de Referência mostrou-se relacionada ao fator $4(p=0,880)$, enquanto a subescala Coordenação Geral estava correlacionada com o fator 9 ( $p=0,641)$. A última subescala Médico Clínico mostrou-se relacionada ao fator $6(p=0,922)$.

Percebe-se, portanto, que, excetuando-se o fator 7 , relacionado a duas subescalas, Disponibilidade do Psiquiatra e Disponibilidade do Técnico de Referência, e o fator 8, que não se destaca em nenhuma correlação, as demais correlações apontam para uma correspondência entre a estrutura teórica da escala e os dados coletados. Destaca-se ainda que todas essas correlações foram estatisticamente significativas $(p<0,01)$.

Mantendo-se a classificação proposta por Dancey e Reidy ${ }^{20}$, das nove correlações, quatro foram moderadas $(0,4$ até $0,6)$ e cinco foram fortes $(>0,7)$ (Tabela 5 ).

Após verificar a correlação entre os fatores e as subescalas, foi realizada a análise da estrutura dos fatores. Essa análise aponta quais itens são predominantes em cada fator. Idealmente, os fatores devem ter sua carga oriunda dos mesmos itens que compõem a subescala com a qual mantêm a mais forte correlação.

A estrutura de fatores encontrada para versão brasileira da escala CONNECT está descrita na Tabela 6. Nesse sentido, os fatores 1 e 2 concentram a carga fatorial dos itens das subescalas Conhecimento do Técnico de Referência e Conhecimento do Psiquiatra, respectivamente.
A subescala Psiquiatra/Técnico de Referência/Profissional do CAPS (itens de 19 a 25) teve quatro de seus sete itens ligados ao fator 3.

Os itens de 26 a 31 da subescala Flexibilidade de Psiquiatra/Técnico de Referência estiveram mais fortemente representados no fator 5 (quatro dos seis itens).

Os itens de 32 a 34 da subescala Coordenação do Psiquiatra/Técnico de Referência estavam todos representados mais fortemente no fator 4 . O mesmo ocorreu com o item 35 da subescala Coordenação Geral, associado ao fator 8, e nos itens 36 e 37 da subescala Médico Clínico, presentes mais fortemente no fator 6

Dos 37 itens investigados, 30 itens foram mais associados aos fatores esperados, dos quais apenas sete itens estavam relacionados a outros fatores (Tabela 6).

\section{DISCUSSÃO}

A ACP confirmatória executada apresenta uma estrutura correspondente à estrutura original da escala, inclusive com grande correspondência entre os itens da subescala e os itens dos fatores gerados na análise.

A força das correlações encontrada entre as subescalas e os fatores da Análise de Componentes Principal foi satisfatória: quatro foram moderadas $(0,4$ até 0,6$)$ e cinco foram fortes $(>0,7)$, todas significativas estatisticamente $(p \leq 0,01)$.

A utilização de ACP para validação de escalas é muito usada na literatura, por ser um método objetivo e reproduzível com boa aplicabilidade. A utilização de um método diferente dos já empregados em validações anteriores desse mesmo instrumento foi recomendada pelos autores da versão em espanhol. As vantagens da ACP tornam essa técnica uma boa solução, pois a validação é tanto mais robusta quanto mais variadas forem as técnicas que reforçam essa validação ${ }^{11,20}$.

Tabela 5. Coeficiente de correlação de Pearson $(p)$ entre as subescalas e os fatores da Análise de Componentes Principais

\begin{tabular}{|c|c|c|c|c|c|c|c|c|c|}
\hline & Fator 1 & Fator 2 & Fator 3 & Fator 4 & Fator 5 & Fator 6 & Fator 7 & Fator 8 & Fator 9 \\
\hline 1. Conhecimento do Psiquiatra & $0,344^{* *}$ & $0,658^{\prime \prime}$ & 0,107 & 0,151 & 0,065 & 0,024 & 0,980 & $-0,025$ & 0,260 \\
\hline 2. Conhecimento do Técnico de Referência & $0,899^{* *}$ & $0,283^{* *}$ & 0,149 & 0,112 & 0,077 & 0,098 & 0,043 & 0,129 & 0,101 \\
\hline 3. Disponibilidade do Psiquiatra & 0,061 & $0,343^{* *}$ & 0,164 & $-0,140$ & 0,096 & 0,101 & $0,748^{\prime \prime}$ & 0,066 & $-0,226^{* *}$ \\
\hline 4. Disponibilidade do Técnico de Referência & $0,414^{* *}$ & $-0,177^{*}$ & $0,304^{* *}$ & $0,207^{*}$ & $0,176^{*}$ & $-0,089$ & $0,561^{*}$ & 0,043 & $0,284^{* *}$ \\
\hline 5. Psiquiatra/Técnico de referência/Profissional do CAPS & $0,183^{*}$ & 0,119 & $0,647^{* *}$ & 0,145 & $-0,002$ & 0,097 & 0,156 & $0,606^{* *}$ & 0,112 \\
\hline 6. Flexibilidade de Psiquiatra/Técnico de Referência & 0,151 & $0,259^{* *}$ & 0,150 & $0,287^{* *}$ & $0,801^{\prime \prime \prime}$ & 0,120 & $-0,004$ & 0,088 & $0,276^{* *}$ \\
\hline 7. Coordenação do Psiquiatra/Técnico de Referência & 0,123 & 0,162 & 0,043 & $0,880^{* *}$ & 0,156 & 0,024 & 0,015 & 0,054 & 0,159 \\
\hline 8. Coordenação Geral & 0,134 & $0,198^{*}$ & $-0,051$ & 0,058 & 0,131 & 0,073 & $-0,037$ & $-0,062$ & $0,641^{* \prime}$ \\
\hline 9. Médico Clínico & 0,133 & $-0,014$ & 0,016 & 0,031 & 0,037 & $0,922^{* *}$ & $-0,034$ & 0,115 & 0,072 \\
\hline
\end{tabular}

Correlaçōes significativas com valor de $p \leq 0,05$ (bicaudal).

"Correlaçōes significativas com valor de $p \leq 0,01$ (bicaudal). 
Tabela 6. Distribuição da carga fatorial dos itens dentre os fatores da análise de Componentes Principais

\begin{tabular}{|c|c|c|c|c|c|c|c|c|c|}
\hline \multirow{2}{*}{ Itens da CONNECT } & \multicolumn{9}{|c|}{ Fatores da Análise de Componentes Principais } \\
\hline & 1 & 2 & 3 & 4 & 5 & 6 & 7 & 8 & 9 \\
\hline \multicolumn{10}{|c|}{ Conhecimento do Psiquiatra } \\
\hline 1 & 0,123 & 0,688 & $-0,066$ & 0,073 & 0,061 & $-0,124$ & 0,056 & 0,029 & 0,085 \\
\hline 2 & 0,154 & 0,716 & 0,191 & $-0,034$ & 0,019 & 0,147 & $-0,062$ & 0,100 & 0,053 \\
\hline 3 & 0,203 & 0,724 & 0,093 & 0,019 & 0,097 & $-0,078$ & 0,177 & 0,181 & 0,112 \\
\hline 4 & 0,251 & 0,653 & 0,039 & 0,074 & $-0,003$ & $-0,104$ & 0,146 & 0,191 & 0,154 \\
\hline 5 & 0,380 & 0,504 & 0,102 & 0,257 & 0,056 & 0,124 & 0,055 & $-0,288$ & $-0,135$ \\
\hline 6 & 0,305 & 0,630 & 0,020 & 0,383 & 0,029 & $-0,018$ & $-0,060$ & $-0,150$ & $-0,156$ \\
\hline \multicolumn{10}{|c|}{ Conhecimento do Técnico de Referência } \\
\hline 7 & 0,690 & 0,284 & $-0,005$ & 0,092 & 0,086 & 0,058 & 0,016 & 0,266 & ,233 \\
\hline 8 & 0,609 & 0,263 & 0,111 & 0,144 & 0,065 & 0,192 & $-0,015$ & 0,199 &, 220 \\
\hline 9 & 0,713 & 0,325 & 0,114 & $-0,002$ & 0,053 & $-0,027$ & $-0,026$ & 0,181 & 135 \\
\hline 10 & 0,676 & 0,172 & 0,076 & 0,198 & 0,056 & 0,068 & 0,185 & $-0,085$ &,- 073 \\
\hline 11 & 0,796 & 0,055 & 0,132 & 0,048 & 0,050 & 0,043 & 0,033 & $-0,050$ &,- 005 \\
\hline 12 & 0,598 & 0,194 & 0,223 & 0,037 & 0,042 & 0,104 & 0,013 & 0,080 &,- 037 \\
\hline \multicolumn{10}{|c|}{ Disponibilidade do Psiquiatra } \\
\hline 13 & 0,101 & 0,367 & 0,578 & $-0,182$ & 0,030 & 0,204 & 0,224 &,- 036 &,- 142 \\
\hline 14 & 0,106 & 0,104 & $-0,131$ & $-0,075$ & 0,222 & 0,091 & 0,622 &,- 008 &,- 337 \\
\hline 15 & $-0,082$ & 0,262 & $-0,043$ & $-0,041$ & $-0,075$ & $-0,079$ & 0,657 & ,179 &, 035 \\
\hline \multicolumn{10}{|c|}{ Disponibilidade do Técnico de Referência } \\
\hline 16 & 0,285 & $-0,230$ & 0,396 & 0,190 & 0,218 & $-0,129$ & 0,334 &,- 008 & ,126 \\
\hline 17 & 0,389 & $-0,065$ & 0,078 & 0,079 & 0,231 & $-0,048$ & 0,345 &,- 011 & ,295 \\
\hline 18 & 0,259 & $-0,126$ & 0,248 & 0,204 & $-0,030$ & $-0,039$ & 0,569 & ,106 & ,204 \\
\hline \multicolumn{10}{|c|}{ Psiquiatra/Técnico de Referência/Profissional do CAPS } \\
\hline 19 & 0,205 & 0,036 & 0,758 & 0,004 & 0,171 & $-0,049$ & $-0,080$ & 0,069 & $-0,022$ \\
\hline 20 & 0,106 & 0,040 & 0,766 & $-0,012$ & $-0,091$ & $-0,012$ & $-0,177$ & 0,171 & 0,116 \\
\hline 21 & $-0,029$ & 0,000 & 0,261 & $-0,035$ & $-0,328$ & 0,044 & 0,359 & 0,074 & 0,489 \\
\hline 22 & 0,202 & 0,118 & 0,480 & 0,312 & $-0,138$ & 0,097 & $-0,051$ & 0,315 & $-0,051$ \\
\hline 23 & 0,134 & 0,091 & 0,095 & 0,099 & $-0,002$ & 0,090 & 0,062 & 0,748 & 0,053 \\
\hline 24 & 0,063 & 0,100 & 0,648 & 0,128 & 0,074 & 0,009 & 0,269 & $-0,061$ & 0,118 \\
\hline 25 & 0,071 & 0,064 & 0,100 & 0,022 & 0,204 & 0,126 & 0,164 & 0,711 & $-0,101$ \\
\hline \multicolumn{10}{|c|}{ Flexibilidade de Psiquiatra/Técnico de Referência } \\
\hline 26 & 0,191 & 0,218 & 0,142 & 0,388 & 0,227 & 0,201 & 0,003 & 0,064 & 0,487 \\
\hline 27 & 0,106 & 0,016 & 0,137 & 0,271 & 0,645 & $-0,230$ & $-0,144$ & 0,271 & 0,153 \\
\hline 28 & 0,170 & $-0,048$ & 0,185 & 0,188 & 0,701 & $-0,168$ & 0,063 & 0,143 & 0,242 \\
\hline 29 & 0,052 & 0,560 & 0,132 & 0,248 & 0,186 & 0,289 & $-0,073$ & $-0,108$ & 0,188 \\
\hline 30 & 0,060 & 0,110 & $-0,055$ & 0,065 & 0,620 & 0,072 & 0,038 & $-0,010$ & $-0,097$ \\
\hline 31 & 0,005 & 0,147 & 0,015 & $-0,047$ & 0,682 & 0,297 & 0,094 & $-0,026$ & 0,064 \\
\hline \multicolumn{10}{|c|}{ Coordenação do Psiquiatra/Técnico de Referência } \\
\hline 32 & 0,151 & 0,147 & 0,063 & 0,749 & 0,064 & $-0,078$ & 0,110 & 0,083 & 0,056 \\
\hline 33 & 0,092 & 0,037 & $-0,011$ & 0,778 & 0,091 & 0,095 & 0,017 & 0,085 & $-0,004$ \\
\hline 34 & 0,043 & 0,218 & 0,053 & 0,559 & 0,240 & 0,047 & $-0,116$ & $-0,057$ & 0,372 \\
\hline \multicolumn{10}{|c|}{ Coordenação Geral } \\
\hline 35 & 0,134 & 0,198 & $-0,051$ & 0,058 & 0,131 & 0,073 & $-0,037$ & $-0,062$ & 0,641 \\
\hline \multicolumn{10}{|c|}{ Médico Clínico } \\
\hline 36 & 0,139 & 0,063 &, 025 & $-0,044$ & 0,051 & 0,847 & $-0,066$ & 0,079 & 0,091 \\
\hline 37 & 0,099 & $-0,110$ &, 002 & 0,121 & 0,011 & 0,831 & 0,015 & 0,138 & 0,033 \\
\hline
\end{tabular}


O intervalo de Correlação Intraclasse das nove subescalas apresentou valores ótimos, com apenas duas subescalas apresentando correlação moderada e todas as demais apresentando valores de ICC entre bom e quase perfeito (> 60).

Não foi objetivo deste estudo analisar a continuidade do cuidado nessa amostra. Entretanto, a pontuação das subescalas é importante para verificar a coerência entre elas. Podemos observar que foi obtida maior pontuação na subescala Coordenação Geral (89,83\%), seguida da subescala Transição Internação - Ambulatório (83,20\%). A menor pontuação foi encontrada na subescala Disponibilidade do Psiquiatra (20,78\%), e a subescala Disponibilidade do Técnico de Referência também apresentou uma pontuação baixa $(34,08 \%)$. Esses resultados, notadamente baixos, também foram encontrados nos grupos da versão em espanhol, tanto no Texas - Disponibilidade do Psiquiatra (16,19\%) e Disponibilidade do Técnico de Referência (31,82\%) - quanto em Porto Rico - Disponibilidade do Psiquiatra (9,96\%) e Disponibilidade do Técnico de Referência (14,77\%). O mesmo foi encontrado no artigo de elaboração da versão original da escala Disponibilidade do Psiquiatra (37,40\%) e Disponibilidade do Técnico de Referência $(41,40 \%)^{10,11}$.

Chavez et al."1 apresentaram duas justificativas para esses baixos valores. A primeira está no fato de que os usuários estejam identificando uma grave limitação dos serviços de saúde mental nos locais pesquisados. Outra justificativa apontada seria uma possível limitação, com perda de equivalência da versão em espanhol.

Como nossos resultados foram semelhantes, entre as duas justificativas apontadas é possível que os nossos resultados reforcem a primeira justificativa, ou seja, tanto o psiquiatra quanto o técnico de referência não estejam suficientemente disponíveis para contatos extraconsultas.

Outro achado relevante do estudo é que não foi encontrada uma forte correlação entre as subescalas e o instrumento completo, da mesma forma que no estudo original da escala ${ }^{10}$. Uma forte correlação entre as subescalas poderia indicar redundância destas.

O item 40 - "Você recebeu a medicação para tomar em casa?" - apresentou uma correlação baixa e não significativa. O que não foi encontrado no estudo de validação da versão em espanhol ${ }^{11}$. Uma possível explicação para isso talvez seja o fato de que alguns pacientes, em vez de receberem a medicação para tomar em casa, são orientados a tomá-la sob supervisão dos profissionais dos CAPS. Isso é particularmente verdade logo após receberem alta da internação, como era o caso dos respondentes desse item. Uma possível redação que contemple essa situação, como, por exemplo, "Você recebeu medicação para tomar em casa ou no CAPS?", deverá ser verificada em novos estudos.

A taxa de adesão dos pacientes à pesquisa foi bastante alta, e a maioria interessou-se em participar após o convite realizado pelos pesquisadores. Destacaram-se positivamente as estratégias adotadas de não interferir na rotina das atividades do paciente e de idas exaustivas aos locais de pesquisa. Os usuários em geral conseguiram responder bem às entrevistas, e em alguns casos foram necessárias explicações adicionais dadas pelos pesquisadores.

Até o momento, a CONNECT é o único instrumento de avaliação da continuidade do cuidado que está adaptado ao contexto brasileiro. Um aspecto relevante do instrumento é o fato de que não se destina ao autopreenchimento, ou seja, deve ser aplicado por um entrevistador diretamente. Isso possibilita que seja aplicado a indivíduos que não saibam ou não possam ler. Por outro lado, implica custos maiores de aplicação e diminui a sensação de anonimato dos entrevistados.

A validação da escala CONNECT é um passo importante no esforço de disponibilizar aos pesquisadores brasileiros mais um instrumento para a realização de pesquisas na área de saúde mental. Além disso, fornece aos profissionais e gestores uma ferramenta útil para a avaliação do tratamento e do serviço.

\section{CONCLUSÃO}

Os resultados obtidos neste estudo mostram que a versão brasileira da escala CONNECT pode ser utilizada com segurança. Espera-se que não só pesquisadores da área, mas, principalmente, gestores em saúde possam utilizar-se desse instrumento para comparar serviços de atenção psicossocial, verificar melhora/piora da continuidade proporcionada pelo serviço e medir o impacto de novas tecnologias, tratamentos ou estruturas disponibilizadas a essa população.

\section{CONTRIBUIÇÕES INDIVIDUAIS}

Diego de Lima Fonseca - Participou da concepção e do desenho do estudo, da coleta de dados, da análise e interpretação dos dados, da elaboração do artigo e aprovou sua versão final.

Michelli Melo Grama - Participou da concepção e desenho do estudo, da coleta de dados, da interpretação dos dados, da elaboração do artigo e aprovou sua versão final.

Giovanni Marcos Lovisi - Participou da concepção e desenho do estudo, da análise e interpretação dos dados, da elaboração do artigo e aprovou sua versão final.

Lúcia Abelha - Participou da concepção e desenho do estudo, da análise e interpretação dos dados, da elaboração do artigo e aprovou sua versão final. 


\section{CONFLITOS DE INTERESSE}

Todos os autores informam não possuir conflitos de interesse a serem declarados.

\section{REFERÊNCIAS}

1. Amarante P (coord.). Loucos pela vida: a trajetória da reforma da assistência psiquiátrica no Brasil. 2. ed. rev. e ampl. Rio de Janeiro: Fiocruz, 2010. p. 136.

2. Brasil. Ministério da Saúde. Secretaria de Atenção à Saúde. Departamento de Ações Programáticas Estratégicas. Coordenação Geral de Saúde Mental, Álcool e outras Drogas. Saúde Mental no SUS: as novas fronteiras da Reforma Psiquiátrica. Relatório de Gestão 2007-2010. Brasilia, DF, jan. 2011. p. 106.

3. Vidal CEL, Bandeira M, Gontijo ED. Reforma da assistência psiquiátrica e serviços residenciais terapêuticos. J Bras Psiquiatr. 2008;57(1):70-9.

4. Lima LA, Legay LF, Lovisi G. 0 processo de reforma psiquiátrica brasileiro na era da globalização: desafios e perspectivas. Cad Saúde Colet. 2004;12(1):9-25.

5. Souza, F. M. et al. Acompanhamento de pacientes do espectro esquizofrênico no território: adaptação da intervenção para períodos de transição ("Critical Time Intervention") para o contexto brasileiro em Centros de Atenção Psicossocial do município do Rio de Janeiro. Cad Saúde Colet. 2012;20(4):427-35.

6. Bachrach LL. Continuity of care for chronic mental patients: a conceptual analysis. Am J Psychiatry. 1981;138(11):1449-56.

7. Tessler RC, Willis G, Gubman GD. Defining and measuring continuity of care. Psychiatr Rehabil J. 1986;10(1):27-38.

8. Test MA. Continuity of care in community treatment. New Directions for Mental Health Services. 1979;2:15-23.
9. Joyce AS, Wild TC, Adair CE, MCDougall GM, Gordon A, Costigan N, Beckie A, Kowalsky L, Pasmeny G, Barnes F. Continuity of care in mental health services: toward clarifying the construct. Can J Psychiatry. 2004;49(8):539-50.

10. Ware NC, Dickey B, Tugenberg T, McHorney CA. CONNECT: a measure of continuity of care in mental health services. Ment Health Serv Res. 2003;5(4):209-21.

11. Chavez LM, Canino G, Shrout PE, Barrio C, Ware NC. Psychometric evaluation of the Spanish version of CONNECT: a measure of continuity of care in mental health services. Int J Methods Psychiatr Res. 2007;16(1):23-33.

12. Duarte CS, Bordin IAS. Instrumentos de avaliação. Rev Bras Psiquiatr. 2000;22(Supl. 2):55-8.

13. Amarante P. Saúde mental e atenção psicossocial. Rio de Janeiro: Fiocruz, 2007. p. 120.

14. Cavalcanti MT, Carvalho MCA, Valência E, Dahl CM, Souza FM. Adaptação da "Critical Time Intervention" para o contexto brasileiro e sua implementação junto a usuários dos Centros de Atenção Psicossocial do município do Rio de Janeiro. Ciênc Saúde Coletiva. 2011;16(12):4635-42.

15. Ribeiro SL. A criação do Centro de Atenção Psicossocial Espaço Vivo. Psicol Cienc Prof. 2004;24(3):92-9.

16. Grama MM. CONNECT: uma medida de continuidade do cuidado em serviços de saúde mental: adaptação transcultural e confiabilidade da escala. 2014. 136 f. Dissertação (Mestrado em Saúde Coletiva) - Centro de Ciências da Saúde, Universidade do Rio de Janeiro, Rio de Janeiro, 2014.

17. Rodrigues DR. Desenho e implantação de um sistema de informação para o serviço de saúde mental no município de Guapimirim. 2013. 70 f. Dissertação (Mestrado em Ciências) - Escola Nacional de Saúde Pública Sergio Arouca, Fundação Oswaldo Cruz, Rio de Janeiro, 2013.

18. McdowelL I. Measuring health: a guide to rating scales and questionnaires. New York: Oxford University Press, 2006.

19. Cohen J. Statistical power analysis for the behavioral sciences Hillsdale, NJ: Erlbaum, 1988.

20. Dancey C, Reidy J. Estatística sem matemática para psicologia: usando SPSS para Windows. Porto Alegre: Artmed, 2006. 